\title{
Beyond the kidney: Nephrologists in the treatment of severe chronic pruritus failing to respond to systemic treatments
}

\author{
Codina S, Sanchez R, Sandoval D, Quero M, Rama I, Cruzado JM and Hueso M* \\ Department of Nephrology, Hospital Universitari Bellvitge, and Bellvitge Research Institute (IDIBELL), Spain
}

\begin{abstract}
We report a case of severe chronic pruritus in a patient with cholestasic liver disease due to a cholangiocarcinoma that did not respond to sequential treatment with bile acid sequestrants, enzyme-inducing agents, opioid antagonists or selective serotonin inhibitors. Since the cause of severe pruritus is the accumulation of some substances in plasma and other tissues, as a result of cholestasis, it has been suggested that the removal of these substances would improve the quality of life of patients. However, the specific nature of the substances and the mechanisms by which they cause itching is still unknown, meaning that there is no targeted therapy or any guidelines available to tackle this situation. Therefore, therapies for chronic pruritus are largely based on clinical experience and anecdotal reports. Considering that Nephrologists are well trained to perform extracorporeal blood purification treatments, we were requested for an experimental therapy with total plasma exchange (TPE), an invasive therapy that has been claimed as effective. However, this report illustrates a case in which TPE displayed a low clinical benefit and prompted us to review the evidence behind the use of extracorporeal devices in cholestatic liver disease.
\end{abstract}

\section{Introduction}

Chronic pruritus, defined as itch persisting for more than 6 weeks, is often associated with poor quality of life [1-3]. The mechanisms underlying chronic pruritus are complex and only partially understood with multiple mediators involved [4-6]. Given the paucity of data from randomized trials, therapeutic choices are largely based on clinical experience and anecdotal reports.

The pathogenesis of pruritus secondary to cholestatic liver disease has been associated with multiple plasma factors such as neurotransmitters (e.g. histamine, serotonin), bile salts, endogenous opioids, progesterone metabolites or lysophosphatidic acid (LPA) [6,7], but there is no correlation with the severity of the underlying disease and liver structure [8]. Thus, the treatment of cholestasis pruritus is currently based on (i) elimination of mediators of pruritus from the enterohepatic circulation with the use of resins or aspiration with a nasogastric tube, (ii) induction of the metabolism of pruritus mediators in the liver with, for example, rifampicin, and (iii) modulation of the central sedation pathway of pruritus with opioid antagonists or with selective inhibitors of serotonin re-uptake [9]. Intractable pruritus is defined by failure on symptoms control despite the sequential regimen with bile acid sequestrants (cholestiramine or clesevelam), enzyme-inducing agents (rifampicin), opioid antagonists (naltrexone) and selective serotonin inhibitors (sertraline) [8]. Currently, correct response to treatment is commonly measured by a visual analogue scale (VAS) or numeric ranged scales (NRS) [10].

In cases with intractable pruritus, an extracorporeal device with adsorbents for removal of protein-bound toxic substances, such as charcoal hemoperfusion, has been claimed as an option [11]. Molecular Adsorbents Recirculation System (MARS) based on albumin dialysis and containing charcoal in the adsorbent circuit has been also used [12]. The simplest extracorporeal approach and one which has also shown to be effective in reducing the severity of pruritus is plasmapheresis (aphaeresis procedure that separates and removes the plasma component from a patient) or Therapeutic Plasma Exchange (when plasmapheresis is followed by replacement with fresh frozen plasma infusion). Thus, American Guidelines on the Use of Apheresis in Clinical practice [13] included "pruritus due to hepatobilliary diseases" to the list of disease that would benefit of using plasmapheresis (PE) or Therapeutic Plasma Exchange (TPE). However, the quality of the evidence is scarce (category III) and may be not applicable to all patients (Recommendation 1C).

Nephrologists have broad experience in the management of extracorporeal blood purification treatment including vascular access, anticoagulation, volume management and prescription for solute clearance. Thus, we were requested to perform a TPE by membrane plasma separation in a patient with refractory chronic pruritus secondary to cholestatic cholangiocarcinoma.

\section{Clinical case}

A 64-year-old male patient with dyslipidemia and benign prostatic hyperplasia presented a hiliar cholangiocarcinoma (Klatskin tumor) that was resected without complications. Few days later after surgery, ascites and hepatic insufficiency appeared. On the tenth day after surgery, mucosal jaundice was observed (Total bilirubin $=167$ $\mu \mathrm{mol} / \mathrm{L}$ ), and 14 days after, moderated pruritus was developed (Total

*Correspondence to: Hueso M, Department of Nephrology, Hospital Universitari Bellvitge, and Bellvitge Research Institute (IDIBELL), Spain, Tel: 34932607602 ; Fax: 34932607603; E-mail: mhueso@idibell.cat

Received: September 14, 2018; Accepted: September 25, 2018; Published: September 27, 2018 
bilirubin $=203 \mu \mathrm{mol} / \mathrm{L})$. Sequential treatment with ursodeoxycholic acid, rifaximin, fenobarbital, naltrexone and topical therapy with calamine and triamcinolone was initiated (Figure 1). The severity of pruritus increased and was graded as 8 using a 10-point numerical rating score (NRS) which lead patient to a moderated anxiety and sleep deprivation (bilirubin $=426 \mu \mathrm{mol} / \mathrm{L}$ ). Since there was no relief of pruritus with the sequential therapy, the use of extracorporeal devices therapy was proposed.

Patient underwent 7 sessions of TPE by membrane plasma separation using a right internal jugular intravenous access. Informed consent was obtained from the patient prior to initiating TPE. All procedures were performed using the PrismafleX Apheresis System with a polypropylene hollow fiber prisma filter (PRISMAFLEX TPE 2000 setTM). In each session, we provided 1 plasma exchange with 2000 $\mathrm{mL}$ of $5 \%$ albumin and $1000 \mathrm{~mL}$ of frozen fresh plasma as replacement fluids. The procedures were performed in 3 consecutive days (at 56, 57, 58 days after admission) and 4 more treatments on alternate days (at 60, 62, 64 and 66 days after hospital admission). During those days, medical treatment for pruritus was continued (Figure 1). Night resting, jaundice, and scratch lesions were assessed daily. Blood tests to monitory bilirubin, histamine, alanine aminotransferase (ALT), aspartate aminotransferase (AST), alkaline phosphatase, $\gamma$-glutamyltransferase (GGT), Urea and histamine were analyzed before each TPE (Table 1). There were no incidents during the TPE sessions.

After 7 sessions of TPE, patient improved jaundice and bilirubin decreased to $211 \mu \mathrm{mol} / \mathrm{L}$. Pruritus initially decreased only to a 7 NRS grade, allowing night resting for two days (between 1st and 3rd procedure), but pruritus rebound on the third day despite sustained systemic treatment. After the 7 procedures, the pruritus was as intense as before the initiation of extracorporeal treatment (measured with a grade 8 NRS) and TPE was stopped and jugular venous catheter was removed.

During 8 months follow-up, several infectious complications occurred despite patient did not receive any chemotherapy or immunosuppressive treatment. Two Spontaneous Bacterial Peritonitis (SBP) appeared, one by Enterococcus faecium after 71 days after the end of TPE (treated with Teicoplanin) and second by Pseudomona aeruginosa after 156 days after the end of TPE. Finally, itching was ameliorating slowly under sustained medical treatment with bile acid sequestrants, enzyme-inducing agents, opioid antagonists or selective serotonin inhibitors (Figure 1), and 87 days after admission patient pruritus disappeared (Bilirubin $=302 / \mu \mathrm{mol} / \mathrm{L}$; NRS 0).

Patient died after 260 days of follow-up due to blood infection by Pseudomona aeruginosa.

\section{Discussion}

This case report illustrates the treatment failure of chronic severe pruritus with TPE in a patient with a cholestastic liver disease. Previously, several reports have suggested their efficacy since 1978, when Geerdink, et al. reported the first case of refractory pruritus successfully treated with PE [14]. Eight years later, Cohen, et al. [15] published the first series of 5 patients with pruritus secondary to primary biliary cirrhosis (PBC) treated with PE using $1000 \mathrm{ml}$ of normal saline and 5\% albumin 3 times per week (maintenance once or twice a week). In those 5 cases, the response was immediate, decreasing the pruritus intensity grade from 3 to 0 or 1 (scored from 0 to 4 ). Beneficial effect of TPE in cholestatic liver pruritus was claimed after Krawczyk, et al. published a series of 17 consecutive patients in which refractory pruritus significantly decreased according to a 10-point NRS and the effect persisted throughout the 90-day follow-up period [16]. Furthermore, the American Society for Aphaeresis included chronic

Table 1. Main analytic findings before and after TPE treatment

\begin{tabular}{|l|c|c|}
\hline Lab Findings & Before TPE (day 56) & $\begin{array}{c}\text { After TPE } \\
\text { (day 67) }\end{array}$ \\
\hline Creatinine $(\mu \mathrm{mol} / \mathrm{L})$ & 84 & 58 \\
\hline AST $(\mu \mathrm{kat} / \mathrm{L})$ & 3.41 & 4.27 \\
\hline ALT $(\mu \mathrm{kat} / \mathrm{L})$ & 1.26 & 1.81 \\
\hline Albumin $(\mathrm{g} / \mathrm{L})$ & 31 & 40 \\
\hline Total Bilirubin $(\mu \mathrm{mol} / \mathrm{L})$ & 352 & 254 \\
\hline GGT $(\mu \mathrm{kat} / \mathrm{L})$ & 1.48 & 0.96 \\
\hline Alkaline phosphatase $(\mu \mathrm{kat} / \mathrm{L})$ & 2.85 & 1.49 \\
\hline Phosphate $(\mathrm{mmol} / \mathrm{L})$ & 1.1 & 0.77 \\
\hline N-acyl-Histamine $(\mu \mathrm{g} / \mathrm{dl})$ & 0.06 & 0.02 \\
\hline Hemoglobin $(\mathrm{g} / \mathrm{L})$ & 106 & 102 \\
\hline Reticulocytes $(\mathrm{x} 10 \mathrm{E} 9 / \mathrm{L})$ & 176 & 121 \\
\hline Leucocytes $(\mathrm{x} 10 \mathrm{E} 9 / \mathrm{L})$ & 7.3 & 6.7 \\
\hline Eosinophils $(\mathrm{x} 10 \mathrm{E} 9 / \mathrm{L})$ & 0.81 & 0.1 \\
\hline
\end{tabular}

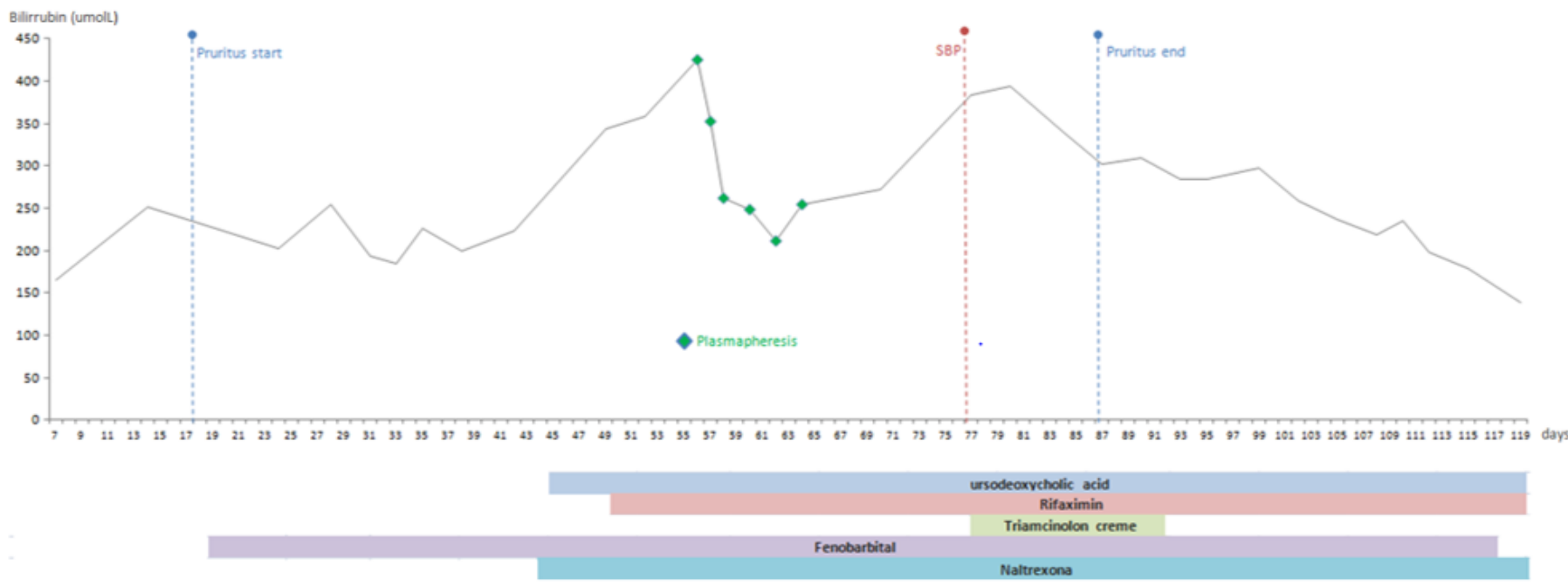

Figure 1. Chronological evolution of Bilirubin levels in the first 100 days after hospital admission. Pruritus starts after 18 days from admission and ends on the $87^{\text {th }}$ day. TPE sessions were performed on days 56,57, 58, 60, 62, 64 and 66. First SBP episode occurred on day 77 
pruritus due to hepatobiliar disease as one of the indications for TPE treatment [13].

Summing up, publications until this date includes 13 reports with 51 patients treated with TPE, associated with an amelioration of pruritus in all but two patients [17]. These two patient's refractories to TPE are part of a series of 26 patients with Benign Recurrent Hepatic Cholestasis [18]. In these series, 6 patients out of the total series required plasmapheresis as a treatment for pruritus with two treatment failures. This article does not describe the characteristics of the procedures.

Thus, why the treatment was failed in our patient? The cause of severe pruritus is not well known, neither which is the metabolite to be eliminated. It has been suggested the role of protein-bound toxic substances and it has been proposed the use of charcoal and other adsorbents [8]. Thus, it has been reported clinical response in 9 out of 15 patients (mostly with PBE) treated with Charcoal hemoperfusion with a median of 5 sessions of 4 hours extracorporeal perfusion per week [11]. In patients with no relief of pruritus despite charcoal hemoperfusion, it has been reported that all but one experienced relief from pruritus when Molecular Adsorbents Recirculation System (MARS) was used (based on albumin dialysis and containing charcoal in the adsorbent circuit refractory). There are experiences with 7 hours treatment for two consecutive days with MARS and with 6 hours treatment for 3 consecutive days, presenting good response with long-lasting effect (ranged from 2 to 5 months) $[12,19]$. Thus, the lack of response could be related to the inefficacy of TPE to remove molecular substances that may be pathogenic. In addition, is the differences observed with our patient related with the dose or the length of the TPE therapy? Most of the cases reported have used albumin 5\%, have exchanged between 1 and 1.3 plasma volumes [20,21], and have used maintained PE on a weekly or monthly basis [22]. However, other causes are plausible.

Are the differences observed with our patient related with what is considered an adequate response? Clinical effectiveness has been measured with different questionnaires, not always validated, and it has been considered an adequate response to treatment both the disappearance and the reduction of symptoms to the category of mild. Some other items like night rest or scratching injuries has been evaluated only in some studies [16]. In most cases, the response is described as "early" although it is not always accurately collected. In the series of 3 cases of Chkheidze, et al. [17], for example, pruritus was considered mild after 5, 7 and 5 sessions respectively. Furthermore, there is a potential positive publication bias in favor of treatment with PE or TPE.

Other differences could contribute to explain the refractoriness to the treatment in our patient. In most cases the primary cholestasic disease was PBC (37 out of 48 cases of PBC, 1 case secondary to pregnancy, 1 case with Primary Sclerosing Cholangitis, 1 case secondary to drugs and the other 6 cases secondary to Benign Recurrent Hepatic Cholestasis) [17]. In our case, the primary cholestatic disease was a malignant cholangiocarcinoma.

The etiopathogenesis of pruritus is complex, multifactorial and not completely understood. In addition, it is known the big role of the affective component associated with this symptomatology. In fact, the use of placebo treatments has shown effective in daily clinical practice [5], and the symptoms of our patient were self-limiting despite the PE treatment was finished.

At last, the decision to stop the treatment after 7 sessions can be considered hasty since at the end of the treatment the levels of bilirubin in blood were still high. However, no clinical response had been observed and no article has managed to significantly correlate levels of bilirubin or bile salts with neither the presence nor the intensity of pruritus.

Finally, we must keep in mind that PE has been associated with an increased risk of infections. However, very few adverse effects are described associated with plasmapheresis in the aforementioned series of cases. It should be noted that the most frequently described adverse effect has been mild urticaria (up to $7.7 \%$ in the series by Shemin, et al. [23] limited with oral medication. In our case, we do not observe any complications related to the TPE treatment since the episodes of SBP occurred, at least, 2 months after the end of treatment. In addition, none of the microorganisms causing infection were encapsulated germs that could be associated with hypogammaglobulinemia secondary to treatment.

\section{Conclusion}

In conclusion, extracorporeal devices and TPE are still an experimental therapy in resistant pruritus due to cholestatic liver diseases accepted in current clinical guidelines. However, the quality of the evidence is still low, and its use should be individualized. This clinical case is an example of the need for a randomized clinical trial to further defend the real effectiveness of plasmapheresis for the treatment of chronic refractory pruritus.

\section{Acknowledgments}

We thank CERCA Program / Generalitat de Catalunya for institutional support.

\section{References}

1. Kini SP, DeLong LK, Veledar E, McKenzie-Brown AM, Schaufele M, et al. (2011) The impact of pruritus on quality of life: the skin equivalent of pain. Arch Dermatol 147: 1153-1156. [Crossref]

2. Mells GF, Pells G, Newton JL, Bathgate AJ, Burroughs AK, et al. (2013) UK-PBC Consortium: Impact of primary biliary cirrhosis on perceived quality of life: the UKPBC national study. Hepatology 58: 273-283.

3. Ständer S, Weisshaar E, Mettang T, Szepietowski JC, Carstens E, et al. (2007) Clinica classification of itch: a position paper of the International Forum for the Study of Itch. Acta Derm Venereol 87: 291-294.

4. Hassan I, Haji ML (2014) Understanding itch: an update on mediators and mechanisms of pruritus. Indian J Dermatol Venereol Leprol 80: 106-114. [Crossref]

5. Kremer AE, Martens JJ, Kulik W, Ruëff F, Kuiper EM, et al. (2010) Lysophosphatidic acid is a potential mediator of cholestatic pruritus. Gastroenterology 139: 1008-1018. [Crossref]

6. Murphy M, Carmichael AJ (2000) Renal itch. Clin Exp Dermatol 25: 103-106 [Crossref]

7. Kremer AE, Namer B, Bolier R, Fischer MJ, Oude Elferink RP, et al. (2015) Pathogenesis and management of pruritus in PBC and PSC. Dig Dis 33: 164-175. [Crossref]

8. Williams R (2007) Extracorporeal devices for treatment of refractory pruritus in cholestatic liver disease. Hepatol Int 11: 371.

9. European Association for the Study of the Liver (2009) EASL clinical practice guidelines: management of cholestatic liver diseases. J Hepatol 51: 237-267.

10. Reich A, Heisig M, Phan NQ, Taneda K, Takamori K, et al. (2012) Visual analogue scale: evaluation of the instrument for the assessment of pruritus. Acta Derm Venereol 92: 497-501. [Crossref]

11. Kittanamongkolchai W, El-Zoghby ZM, Eileen Hay J, Wiesner RH, Kamath PS, et al. (2017) Charcoal hemoperfusion in the treatment of medically refractory pruritus in cholestatic liver disease. Hepatol Int 11: 384.

12. Parés A, Herrera M, Avilés J, Sanz M, Mas A (2010) Treatment of resistant pruritus from cholestasis with albumin dialysis: combined analysis of patients from three centers. J Hepatol 53: 307-312. [Crossref] 
13. Schwartz J, Padmanabhan A, Aqui N, Balogun RA, Conelly-Smith L, et al. (2016) Guidelines on the use of therapeutic apheresis in clinical practice- evidence-based approach from the writing committee of the american society for apheresis. J Clin Apher 31: 149-338.

14. Geerdink P, Snel P, van Berge Henegouwen GP, Huybregts A, Tangerman A, et al. (1978) Treatment of intractable pruritus in patients with cholestatic jaundice by plasma exchange and plasma perfusion. Neth J Med 21: 239-244.

15. Cohen LB, Ambinder EP, Wolke AM, Field SP, Schaffner F (1985) Role of plasmapheresis in primary biliary cirrhosis. Gut 26: 291-294. [Crossref]

16. Krawczyk M, Liebe R, Wasilewicz M, Wunsch E, Raszeja-Wyszomirska J, et al. (2017) Plasmapheresis exerts a long-lasting antipruritic effect in severe cholestatic itch. Liver Int 37: 743-747.

17. Chkheidze R, Joseph R, Burner J, Matevosyan K (2018) Plasma exchange for the management of refractory pruritus of cholestasis: A report of three cases and review of literature. J Clin Apher 33: 412-418. [Crossref]
18. Brenard R, Geubel AP, Benhamou JP (1989) Benign recurrent intrahepatic cholestasis. A report of 26 cases. J Clin Gastroenterol 11: 546-551. [Crossref]

19. Leckie P, Tritto G, Mookerjee R, Davies N, Jones D, et al. (2012) “Out-patient' albumin dialysis for cholestatic patients with intractable pruritus. Aliment Pharmacol Ther 35 : 696-704

20. Kohan AI, Findor JA, Igartua EB, Villaravid N (1991) Intensive plasmapheresis as an alternative therapy for intractable pruritus of primary biliary cirrhosis. Transfus $S c i$ 12:197-200.

21. Warren JE, Blaylock RC, Silver RM (2005) Plasmapheresis for the treatment of intrahepatic cholestasis of pregnancy refractory to medical treatment. Am J Obstet Gynecol 192: 2088-2089.

22. Axelsson CG, Hallback DA (2013) Twenty-six years of plasma ex-change for symptomatic treatment of pruritus in primary biliary cirrhosis. Transfus Apher Sci 49: $652-654$.

23. Shemin D, Briggs D, Greenan M (2007) Complications of therapeutic plasma exchange: a prospective study of 1,727 procedures. J Clin Apher 22: 270-276. [Crossref]

Copyright: ( 2018 Codina S. This is an open-access article distributed under the terms of the Creative Commons Attribution License, which permits unrestricted use, distribution, and reproduction in any medium, provided the original author and source are credited. 